Article

\title{
Determinant Forms, Difference Equations and Zeros of the $q$-Hermite-Appell Polynomials
}

\author{
Subuhi Khan * and Tabinda Nahid \\ Department of Mathematics, Aligarh Muslim University, Aligarh 202001, India; \\ tabindanahid@gmail.com or tabinda.rs@amu.ac.in \\ * Correspondence: subuhi2006@gmail.com
}

Received: 26 October 2018; Accepted: 13 November 2018; Published: 17 November 2018

\begin{abstract}
The present paper intends to introduce the hybrid form of $q$-special polynomials, namely $q$-Hermite-Appell polynomials by means of generating function and series definition. Some significant properties of $q$-Hermite-Appell polynomials such as determinant definitions, $q$-recurrence relations and $q$-difference equations are established. Examples providing the corresponding results for certain members belonging to this $q$-Hermite-Appell family are considered. In addition, graphs of certain $q$-special polynomials are demonstrated using computer experiment. Thereafter, distribution of zeros of these $q$-special polynomials is displayed.
\end{abstract}

Keywords: $q$-Appell polynomials; $q$-Hermite polynomials; $q$-Hermite-Appell polynomials; $q$-recurrence relations; $q$-difference equations

\section{Introduction}

In last few decades, a progressive instantaneous development has been found in the field of $q$-calculus because of its fundamental importance in numerous areas such as applied mathematics, mechanics, mathematical physics, Lie theory and quantum algebra, see for example [1-4]. The subject of $q$-calculus began to appear in the nineteenth century because of its applications in several branches of science and engineering. Throughout the present paper, $\mathbb{C}$ indicates the set of complex numbers, $\mathbb{N}$ designates set of natural numbers and $\mathbb{N}_{0}$ designates set of non-negative integers. Further, the variable $q \in \mathbb{C}$ such that $|q|<1$.

We review certain definitions and concepts related to the $q$-calculus from [1], which will be used throughout this work.

- $q$-analogue of shifted factorial $(a)_{n}$ is specified as

$$
(a ; q)_{0}=1,(a ; q)_{n}=\prod_{m=1}^{n}\left(1-q^{m-1} a\right), n \in \mathbb{N} ;(a ; q)_{\infty}=\prod_{m=0}^{\infty}\left(1-q^{m} a\right), \quad a \in \mathbb{C} .
$$

- $q$-analogues of $a \in \mathbb{C}$ is specified as

$$
[a]_{q}=\frac{1-q^{a}}{1-q}, q \in \mathbb{C} \backslash\{1\}
$$

and $q$-factorial function is specified as

$$
[n]_{q} !=\prod_{m=1}^{n}[m]_{q}=\frac{(q ; q)_{n}}{(1-q)^{n}}, q \neq 1 ; n \in \mathbb{N},[0]_{q} !=1 ; 0<q<1 .
$$

- $\quad q$-binomial coefficient $\left[\begin{array}{l}n \\ k\end{array}\right]_{q}$ is specified as 


$$
\left[\begin{array}{l}
n \\
k
\end{array}\right]_{q}=\frac{[n]_{q} !}{[k]_{q} ![n-k]_{q} !}=\frac{(q ; q)_{n}}{(q ; q)_{k}(q ; q)_{n-k}}, \quad k=0,1, \ldots, n, \quad n \in \mathbb{N}_{0}
$$

- $q$-exponential function is specified as

$$
e_{q}(x)=\sum_{n=0}^{\infty} \frac{x^{n}}{[n]_{q} !}:=\frac{1}{((1-q) x ; q)_{\infty}}, \quad|x|<|1-q|^{-1} .
$$

The $q$-analogue of derivative of a function $f$ at a point $0 \neq z \in \mathbb{C}$, denoted by $D_{q} f$ is specified by

$$
D_{q} f(z)=\frac{f(q z)-f(z)}{q z-z} .
$$

For any two arbitrary functions $f(z)$ and $g(z)$, the following relation for $q$-derivative operator $D_{q}$ holds true:

$$
D_{q, z}(f(z) g(z))=f(z) D_{q, z} g(z)+g(q z) D_{q, z} f(z) .
$$

Next, we review the concepts and results related to the $q$-Appell, $q$-Bernoulli, $q$-Euler, $q$-Genochhi and $q$-Hermite polynomials.

In 1880, Appell [5] introduced an indispensable class of polynomial sequences, known as the Appell polynomials. After that, an important generalization of these Appell polynomials is given by Al-Salam [6] and some of its properties are studied. The $q$-Appell polynomials $A_{n, q}(x)$ satisfy the following $q$-difference equation:

$$
D_{q}(x)\left\{A_{n, q}(x)\right\}=[n]_{q} A_{n-1, q}(x), \quad n=0,1,2, \cdots
$$

and have the following generating equation [6]:

$$
A_{q}(t) e_{q}(x t)=\sum_{n=0}^{\infty} A_{n, q}(x) \frac{t^{n}}{[n]_{q} !},
$$

where

$$
A_{q}(t):=\sum_{n=0}^{\infty} A_{n, q} \frac{t^{n}}{[n]_{q} !}, \quad A_{0, q}=1 ; \quad A_{q}(t) \neq 0 .
$$

is an analytic function at $t=0$ and $A_{n, q}:=A_{n, q}(0)$.

The $q$-Bernoulli, $q$-Euler and $q$-Genocchi polynomials perform an essential role in various expansions and approximation formulae, which are useful both in analytic theory of numbers and in classical and numerical analysis. Different members of $q$-Appell family can be obtained by selecting appropriate generating function $A_{q}(t)$. These members along with their notations, names, generating functions and series definitions are listed in Table 1. 
Table 1. Members related to $q$-Appell family.

\begin{tabular}{|c|c|c|c|c|}
\hline S. No. & $A_{q}(t)$ & $\begin{array}{l}\text { Name of } q \text {-Special } \\
\text { Polynomial and } \\
\text { Related Number }\end{array}$ & Generating Function & Series Definition \\
\hline I. & $\left(\frac{t}{e_{q}(t)-1}\right)$ & $\begin{array}{l}\text { q-Bernoulli } \\
\text { polynomials } \\
\text { and numbers }[7,8]\end{array}$ & $\begin{aligned}\left(\frac{t}{e_{q}(t)-1}\right) e_{q}(x t) & =\sum_{n=0}^{\infty} B_{n, q}(x) \frac{t^{n}}{[n]_{q} !} \\
\left(\frac{t}{e_{q}(t)-1}\right) & =\sum_{n=0}^{\infty} B_{n, q} \frac{t^{n}}{[n]_{q} !} \\
B_{n, q} & :=B_{n, q}(0)\end{aligned}$ & $B_{n, q}(x)=\sum_{k=0}^{n}\left[\begin{array}{l}n \\
k\end{array}\right]_{q} B_{k, q} x^{n-k}$ \\
\hline II. & $\left(\frac{2}{e_{q}(t)+1}\right)$ & $\begin{array}{c}\text { q-Euler } \\
\text { polynomials } \\
\text { and numbers }[8,9]\end{array}$ & $\begin{aligned}\left(\frac{2}{e_{q}(t)+1}\right) e_{q}(x t) & =\sum_{n=0}^{\infty} E_{n, q}(x) \frac{t^{n}}{[n]_{q} !} \\
\left(\frac{2}{e_{q}(t)+1}\right) & =\sum_{n=0}^{\infty} E_{n, q} \frac{t^{n}}{[n]_{q} !} \\
E_{n, q} & :=E_{n, q}(0)\end{aligned}$ & $E_{n, q}(x)=\sum_{k=0}^{n}\left[\begin{array}{l}n \\
k\end{array}\right]_{q} E_{k, q} x^{n-k}$ \\
\hline III. & $\left(\frac{2 t}{e_{q}(t)+1}\right)$ & $\begin{array}{c}\text { q-Genocchi } \\
\text { polynomials } \\
\text { and numbers }[9,10]\end{array}$ & $\begin{aligned}\left(\frac{2 t}{e_{q}(t)+1}\right) e_{q}(x t) & =\sum_{n=0}^{\infty} G_{n, q}(x) \frac{t^{n}}{[n]_{q} !} \\
\left(\frac{2 t}{e_{q}(t)+1}\right) & =\sum_{n=0}^{\infty} G_{n, q} \frac{t^{n}}{[n]_{q} !} \\
G_{n, q} & :=G_{n, q}(0)\end{aligned}$ & $G_{n, q}(x)=\sum_{k=0}^{n}\left[\begin{array}{l}n \\
k\end{array}\right]_{q} G_{k, q} x^{n-k}$ \\
\hline
\end{tabular}

The $q$-Bernoulli , $q$-Euler and $q$-Genocchi numbers have deep connections with number theory and occur in combinatorics. The first few values of $B_{n, q}[8], E_{n, q}$ [8] and $G_{n, q}$ [10] are given in Table 2.

Table 2. Values of first five $B_{n, q}, E_{n, q}$ and $G_{n, q}$.

\begin{tabular}{cccccc}
\hline $\boldsymbol{n}$ & $\mathbf{0}$ & $\mathbf{1}$ & $\mathbf{2}$ & $\mathbf{3}$ & $\mathbf{4}$ \\
\hline$B_{n, q}$ & 1 & $-(1+q)^{-1}$ & $q^{2}\left([3]_{q} !\right)^{-1}$ & $(1-q) q^{3}\left([2]_{q}\right)^{-1}\left([4]_{q}\right)^{-1}$ & $q^{4}\left(1-q^{2}-2 q^{3}-q^{4}+q^{6}\right)\left([2]_{q}^{2}[3]_{q}[5]_{q}\right)^{-1}$ \\
\hline$E_{n, q}$ & 1 & $-\frac{1}{2}$ & $\frac{1}{4}(-1+q)$ & $\frac{1}{8}\left(-1+2 q+2 q^{2}-q^{3}\right)$ & $\frac{1}{16}(q-1)[3]_{q} !\left(q^{2}-4 q+1\right)$ \\
\hline$G_{n, q}$ & 0 & $\frac{2-q}{1+q}$ & $\frac{q(q-5)}{(q+1)^{2}}$ & $-\frac{3 q^{2}(q-5)}{(1+q)^{3}}-\frac{3 q(2-q)}{(1+q)^{2}}-\frac{q}{1+q}$ & $\frac{-3 q}{1+q}\left(\frac{3 q^{3}+10 q^{2}-28 q+7}{(1+q)^{3}}\right)$ \\
\hline \multicolumn{7}{c}{$\begin{array}{l}\text { Note: Since } G_{0, q}=0 \Rightarrow \\
E_{n, q}(x) \text { is } n . \text { Hence, } G_{n, q}(x) \text { is considered in the class of polynomial sequences which are not } q \text {-Appell in } \\
\text { strong sense. }\end{array}$}
\end{tabular}

The $q$-Hermite polynomials are special or limiting cases of the orthogonal polynomials as they contains no parameter other than $q$ and appears to be at the bottom of a hierarchy of the classical $q$-orthogonal polynomials [2]. These polynomials are very important as many more complicated families of the orthogonal polynomials can be represented in terms of the $q$-Hermite polynomials $H_{n, q}(x)$. The $H_{n, q}(x)$ possess the following generating relation [11]:

$$
\begin{gathered}
F_{q}(x, t):=F_{q}(t) e_{q}(x t)=\sum_{n=0}^{\infty} H_{n, q}(x) \frac{t^{n}}{[n]_{q} !}, \\
F_{q}(t):=\sum_{n=0}^{\infty}(-1)^{n} q^{n(n-1) / 2} \frac{t^{2 n}}{[2 n]_{q} ! !}, \quad[2 n]_{q} ! !=[2 n]_{q}[2 n-2]_{q} \ldots[2]_{q} .
\end{gathered}
$$

Moreover

$$
\frac{D_{q, t} F_{q}(t)}{F_{q}(q t)}=-t
$$

and

$$
D_{q, x} H_{n, q}(x)=[n]_{q} H_{n-1, q}(x) .
$$

In the present article, a new family of $q$-Hermite-Appell polynomials is introduced and many interesting properties of these $q$-special polynomials are studied. The generating function, series representation and determinant forms for this new family of polynomials are established. 
Further, the $q$-recurrence relations and $q$-difference equations for this family and certain specific $q$-members related to this family are derived. In addition, shapes of this newly introduced $q$-special polynomials are shown graphically and their zeros are also explored using numerical simulations.

\section{2. $q$-Hermite-Appell Polynomials}

This section aims to introduce $q$-Hermite-Appell polynomials ( $q$-HAP) by means of generating function. Further, the series expansion and determinant definition of these $q$-special polynomials are established.

Utilizing expansion (5) in Relation (9) and then replacing powers of $x^{0}, x^{1}, x^{2}, \ldots, x^{n}$ of $x$ by the correlating polynomials $H_{0, q}(x), H_{1, q}(x), \ldots, H_{n, q}(x)$ and after summing up the terms of the resultant equation and indicating resultant $q$-HAP in r.h.s. by ${ }_{H} A_{n, q}(x)$, the following generating relation for $q$-Hermite-Appell polynomials ${ }_{H} A_{n, q}(x)$ is obtained:

$$
G_{q}(x, t):=A_{q}(t) F_{q}(t) e_{q}(x t)=\sum_{n=0}^{\infty}{ }_{H} A_{n, q}(x) \frac{t^{n}}{[n]_{q} !} .
$$

Using Equations (10) and (11) in generating Equation (15), simplifying and comparing the coefficients of identical powers of $t$ in obtained relation, the following series definition of $q$-Hermite-Appell polynomials ${ }_{H} A_{n, q}(x)$ is obtained:

$$
{ }_{H} A_{n, q}(x)=\sum_{k=0}^{n}\left[\begin{array}{l}
n \\
k
\end{array}\right]_{q} A_{k, q} H_{n-k, q}(x) .
$$

Certain members of $q$-Appell family are given in Table 1 . For suitable selections of function $A_{q}(t)$ in generating Relation (15), different members related to $q$-Hermite-Appell family are obtained. These members along with their notations, names, generating functions and series definitions are mentioned in Table 3.

\begin{tabular}{|c|c|c|c|c|}
\hline S. No. & $A_{q}(t)$ & $\begin{array}{l}\text { Notation and } \\
\text { Name of the } \\
\text { Resultant Member }\end{array}$ & Generating Function & Series Definition \\
\hline I. & $\left(\frac{t}{e_{q}(t)-1}\right)$ & $\begin{array}{c}{ }_{H} B_{n, q}(x):= \\
q \text {-Hermite-Bernoulli } \\
\text { polynomials ( } q \text {-HBP) }\end{array}$ & $\begin{array}{l}\left(\frac{t}{e_{q}(t)-1}\right) F_{q}(t) e_{q}(x t) \\
=\sum_{n=0}^{\infty}{ }_{H} B_{n, q}(x) \frac{t^{n}}{[n]_{q} !}\end{array}$ & ${ }_{H} B_{n, q}(x)=\sum_{k=0}^{n}\left[\begin{array}{l}n \\
k\end{array}\right]_{q} B_{k, q} H_{n-k, q}(x)$ \\
\hline II. & $\left(\frac{2}{e_{q}(t)+1}\right)$ & $\begin{array}{c}{ }_{H} E_{n, q}(x):= \\
q \text {-Hermite-Euler } \\
\text { polynomials ( } q \text {-HEP) }\end{array}$ & $\begin{array}{l}\left(\frac{2}{e_{q}(t)+1}\right) F_{q}(t) e_{q}(x t) \\
=\sum_{n=0}^{\infty} H E_{n, q}(x) \frac{t^{n}}{[n]_{q} !}\end{array}$ & ${ }_{H} E_{n, q}(x)=\sum_{k=0}^{n}\left[\begin{array}{l}n \\
k\end{array}\right]_{q} E_{k, q} H_{n-k, q}(x)$ \\
\hline III. & $\left(\frac{2 t}{e_{q}(t)+1}\right)$ & $\begin{array}{c}{ }_{H} G_{n, q}(x):= \\
q \text {-Hermite-Genocchi } \\
\text { polynomials ( } q \text {-HGP) }\end{array}$ & $\begin{array}{l}\left(\frac{2 t}{e_{q}(t)+1}\right) F_{q}(t) e_{q}(x t) \\
=\sum_{n=0}^{\infty} H G_{n, q}(x) \frac{t^{n}}{[n]_{q} !}\end{array}$ & ${ }_{H} G_{n, q}(x)=\sum_{k=0}^{n}\left[\begin{array}{l}n \\
k\end{array}\right]_{q} G_{k, q} H_{n-k, q}(x)$ \\
\hline
\end{tabular}

Table 3. Members related to $q$-Hermite-Appell family.

On taking $x=0$ in Relation (16), the following series definition for $q$-Hermite-Appell numbers $(q-\mathrm{HAN})_{H} A_{n, q}:={ }_{H} A_{n, q}(0)$ is obtained:

$$
{ }_{H} A_{n, q}=\sum_{k=0}^{n}\left[\begin{array}{l}
n \\
k
\end{array}\right]_{q} A_{k, q} H_{n-k, q}
$$


where $H_{n, q}$ denotes $q$-Hermite numbers.

Next, on taking $x=0$ in series definitions of $q$-HBP ${ }_{H} B_{n, q}(x), q-\mathrm{HEP}_{H} E_{n, q}(x)$ and $q$-HGP ${ }_{H} G_{n, q}(x)$ given in Table 3 and in view of notations given in Table 1, the $q$-Hermite-Bernoulli, $q$-Hermite-Euler and $q$-Hermite-Genocchi numbers are obtained. These numbers are listed in Table 4.

Table 4. Numbers Related to Certain Members of $q$-Hermite-Appell Family.

\begin{tabular}{ccc}
\hline S. No. & Notation and name of resultant numbers & Series Definition \\
\hline I. & ${ }_{H} B_{n, q}:={ }_{H} B_{n, q}(0)$ & ${ }_{H} B_{n, q}=\sum_{k=0}^{n}\left[\begin{array}{l}n \\
k\end{array}\right]_{q} B_{k, q} H_{n-k, q}$ \\
& $q$-Hermite-Bernoulli numbers $(q$-HBN $)$ & \\
\hline II. & ${ }_{H} E_{n, q}:={ }_{H} E_{n, q}(0)$ & ${ }_{H} E_{n, q}=\sum_{k=0}^{n}\left[\begin{array}{l}n \\
k\end{array}\right]_{q} E_{k, q} H_{n-k, q}$ \\
& $q$-Hermite-Euler numbers $(q$-HEN $)$ & \\
III. & ${ }_{H} G_{n, q}:={ }_{H} G_{n, q}(0)$ & ${ }_{H} G_{n, q}=\sum_{k=0}^{n}\left[\begin{array}{l}n \\
k\end{array}\right]_{q} G_{k, q} H_{n-k, q}$ \\
& $q$-Hermite-Genocchi numbers $(q$-HGN $)$ & \\
\hline
\end{tabular}

In 1982, several characterization and clarification of $q$-Appell family are provided by Srivastava [12]. During the past few decades, the $q$-Appell polynomials have been studied from various aspects, see for example [3,4] and using numerous techniques in [13]. The determinant form of $q$-Appell polynomials is obtained by Keleshteri and Mahmudov in [14]. Due to the importance of determinant forms for computational and applied purposes, the determinant definitions of $q$-HAP ${ }_{H} A_{n, q}(x)$ together with certain members belonging to this $q$-Hermite-Appell family are obtained. By using a similar approach ([14], p. 359 (Theorem 7)) and in view of Equations (11) and (15), the following determinant form for $q$-HAP ${ }_{H} A_{n, q}(x)$ is obtained:

Definition 1. The $q$-Hermite-Appell polynomials ${ }_{H} A_{n, q}(x)$ of degree $n$ are defined by

$$
\begin{aligned}
& { }_{H} A_{0, q}(x)=\frac{1}{\beta_{0, q}}, \\
& { }_{H} A_{n, q}(x)=\frac{(-1)^{n}}{\left(\beta_{0, q}\right)^{n+1}}\left|\begin{array}{cccccc}
1 & H_{1, q}(x) & H_{2, q}(x) & \cdots & H_{n-1, q}(x) & H_{n, q}(x) \\
\beta_{0, q} & \beta_{1, q} & \beta_{2, q} & \cdots & \beta_{n-1, q} & \beta_{n, q} \\
0 & \beta_{0, q} & {\left[\begin{array}{l}
2 \\
1
\end{array}\right]_{q} \beta_{1, q}} & \cdots & {\left[\begin{array}{c}
n-1 \\
1
\end{array}\right]_{q} \beta_{n-2, q}} & {\left[\begin{array}{c}
n \\
1
\end{array}\right]_{q} \beta_{n-1, q}} \\
0 & 0 & \beta_{0, q} & \cdots & {\left[\begin{array}{c}
n-1 \\
2
\end{array}\right]_{q} \beta_{n-3, q}} & {\left[\begin{array}{c}
n \\
2
\end{array}\right]_{q} \beta_{n-2, q}} \\
\vdots & \vdots & \vdots & \ddots & \vdots & \vdots \\
0 & 0 & 0 & \cdots & \beta_{0, q} & {\left[\begin{array}{c}
n \\
n-1
\end{array}\right]_{q} \beta_{1, q}}
\end{array}\right|,
\end{aligned}
$$

where $n=1,2, \ldots$ and $H_{n, q}(x)(n=1,2, \ldots)$ are $q$-Hermite polynomials; $\beta_{0, q} \neq 0$ and

$$
\begin{aligned}
& \beta_{0, q}=\frac{1}{A_{0, q}} \\
& \beta_{n, q}=-\frac{1}{A_{0, q}}\left(\sum_{k=1}^{n}\left[\begin{array}{l}
n \\
k
\end{array}\right]_{q} A_{k, q} \beta_{n-k, q}\right), \quad n=1,2, \ldots .
\end{aligned}
$$

Remark 1. Since the $q-H B P{ }_{H} B_{n, q}(x), q-H E P{ }_{H} E_{n, q}(x)$ and $q-H G P{ }_{H} G_{n, q}(x)$ mentioned in Table 3 are particular members of $q$-Hermite-Appell family ${ }_{H} A_{n, q}(x)$. Thus, by making suitable choices for the coefficients $\beta_{0, q}$ and $\beta_{i, q}(i=1,2, \ldots, n)$ in determinant definition of $q$-HAP ${ }_{H} A_{n, q}(x)$, the determinant definitions of $q$-HBP ${ }_{H} B_{n, q}(x), q-H E P{ }_{H} E_{n, q}(x)$ and $q-H G P{ }_{H} G_{n, q}(x)$ can be obtained. For instance, 
taking $\beta_{0, q}=1, \beta_{i, q}=\frac{1}{[i+1]_{q}}(i=1,2, \cdots, n)$ in Equation (18), the following determinant form of $q$-HBP ${ }_{H} B_{n, q}(x)$ is obtained:

Definition 2. The $q$-Hermite-Bernoulli polynomials ${ }_{H} B_{n, q}(x)$ of degree $n$ are defined by

$$
\begin{aligned}
& { }_{H} B_{0, q}(x)=1 \text {, } \\
& { }_{H} B_{n, q}(x)=(-1)^{n}\left|\begin{array}{cccccc}
1 & H_{1, q}(x) & H_{2, q}(x) & \cdots & H_{n-1, q}(x) & H_{n, q}(x) \\
1 & \frac{1}{[2]_{q}} & \frac{1}{[3]_{q}} & \cdots & \frac{1}{[n]_{q}} & \frac{1}{[n+1]_{q}} \\
0 & 1 & {\left[\begin{array}{c}
2 \\
1
\end{array}\right]_{q} \frac{1}{[2]_{q}}} & \cdots & {\left[\begin{array}{c}
n-1 \\
1
\end{array}\right]_{q} \frac{1}{[n-1]_{q}}} & {\left[\begin{array}{c}
n \\
1
\end{array}\right]_{q} \frac{1}{[n]_{q}}} \\
0 & 0 & 1 & \cdots & {\left[\begin{array}{c}
n-1 \\
2
\end{array}\right]_{q} \frac{1}{[n-2]_{q}}} & {\left[\begin{array}{c}
n \\
2
\end{array}\right]_{q} \frac{1}{[n-1]_{q}}} \\
\vdots & \vdots & \vdots & \ddots & \vdots & \vdots \\
0 & 0 & 0 & \cdots & 1 & {\left[\begin{array}{c}
n \\
n-1
\end{array}\right]_{q} \frac{1}{[2]_{q}}}
\end{array}\right|, n=1,2, \cdots,
\end{aligned}
$$

where $H_{n, q}(x)(n=1,2, \cdots)$ are $q$-Hermite polynomials.

Next, taking $\beta_{0, q}=1$ and $\beta_{i, q}=\frac{1}{2}(i=1,2, \cdots, n)$ in Equation (18), the following determinant definition of $q$-HEP ${ }_{H} E_{n, q}(x)$ is obtained:

Definition 3. The $q$-Hermite-Euler polynomials ${ }_{H} E_{n, q}(x)$ of degree $n$ are defined by

$$
{ }_{H} E_{0, q}(x)=1, \quad\left|\begin{array}{cccccc}
1 & H_{1, q}(x) & H_{2, q}(x) & \cdots & H_{n-1, q}(x) & H_{n, q}(x) \\
1 & \frac{1}{2} & \frac{1}{2} & \cdots & \frac{1}{2} & \frac{1}{2} \\
0 & 1 & {\left[\begin{array}{l}
2 \\
1
\end{array}\right]_{q} \frac{1}{2}} & \cdots & {\left[\begin{array}{c}
n-1 \\
1
\end{array}\right]_{q} \frac{1}{2}} & {\left[\begin{array}{c}
n \\
1
\end{array}\right]_{q} \frac{1}{2}} \\
0 & 0 & 1 & \cdots & {\left[\begin{array}{c}
n-1 \\
2
\end{array}\right]_{q} \frac{1}{2}} & {\left[\begin{array}{c}
n \\
2
\end{array}\right]_{q} \frac{1}{2}} \\
\vdots & \vdots & \vdots & \ddots & \vdots & \vdots \\
0 & 0 & 0 & \cdots & 1 & {\left[\begin{array}{c}
n \\
n-1
\end{array}\right]_{q} \frac{1}{2}}
\end{array}\right|, n=1,2, \cdots,
$$

where $H_{n, q}(x)(n=1,2, \cdots)$ are $q$-Hermite polynomials.

Further, taking $\beta_{0, q}=1$ and $\beta_{i, q}=\frac{1}{2[i+1]_{q}}(i=1,2, \cdots, n)$ in Equation (18), the following determinant definition of $q$-HGP ${ }_{H} G_{n, q}(x)$ is obtained: 
Definition 4. The q-Hermite-Genocchi polynomials ${ }_{H} G_{n, q}(x)$ of degree $n$ are defined by

$$
{ }_{H} G_{0, q}(x)=1, \quad\left|\begin{array}{cccccc}
1 & H_{1, q}(x) & H_{2, q}(x) & \cdots & H_{n-1, q}(x) & H_{n, q}(x) \\
1 & \frac{1}{2[2]_{q}} & \frac{1}{2[3]_{q}} & \cdots & \frac{1}{2[n]_{q}} & \frac{1}{2[n+1]_{q}} \\
0 & 1 & {\left[\begin{array}{ccccc}
2 \\
1
\end{array}\right]_{q, q} \frac{1}{2[2]_{q}}} & \cdots & {\left[\begin{array}{c}
n-1 \\
1
\end{array}\right]_{q} \frac{1}{2[n-1]_{q}}} & {\left[\begin{array}{c}
n \\
1
\end{array}\right]_{q} \frac{1}{2[n]_{q}}} \\
0 & 0 & 1 & \cdots & {\left[\begin{array}{c}
n-1 \\
2
\end{array}\right]_{q} \frac{1}{2[n-2]_{q}}} & {\left[\begin{array}{c}
n \\
2
\end{array}\right]_{q} \frac{1}{2[n-1]_{q}}} \\
\vdots & \vdots & \vdots & \ddots & \vdots & \vdots \\
0 & 0 & 0 & \cdots & 1 & {\left[\begin{array}{c}
n \\
n-1
\end{array}\right]_{q} \frac{1}{2[2]_{q}}}
\end{array}\right|, n=1,2, \cdots
$$

where $H_{n, q}(x)(n=1,2, \cdots)$ are $q$-Hermite polynomials.

Remark 2. The determinant definitions of $q-H B N_{H} B_{n, q}, q-H E N_{H} E_{n, q}$ and $q-H G N_{H} G_{n, q}$ can be obtained by taking $x=0$ in Equations (20)-(22) respectively and then using suitable notations from Table 4 (I-III).

In the next section, $q$-recurrence relations and $q$-difference equations of $q$-HAP ${ }_{H} A_{n, q}(x)$ and also for certain members belonging to this family are derived.

\section{3. $q$-Recurrence Relations and $q$-Difference Equations}

The differential or difference equations, linear second order $q$-difference equations are of particular interest in the theory and applications of $q$-difference equations. In 2002, differential equation involving Appell polynomials is studied by He and Ricci [15] by making use of factorization method. Later in 2014, Mahmudov [11] applied an innovative technique to obtain $q$-recurrence relations and $q$-difference equations for $q$-Appell polynomials $A_{n, q}(x)$ together with certain members using only lowering operators that are $q$-derivatives.

The $q$-recurrence relation for $q$-HAP ${ }_{H} A_{n, q}(x)$ is established in the form of the following result:

Theorem 1. The following linear homogeneous q-recurrence relations for q-Hermite-Appell polynomials ${ }_{H} A_{n, q}(x)$ holds true:

$$
\begin{aligned}
{ }_{H} A_{n, q}(q x) & =\frac{1}{[n]_{q}} \alpha_{0} q^{n}{ }_{H} A_{n, q}(x)+q^{n}\left(x+\alpha_{1} q^{-1}\right)_{H} A_{n-1, q}(x) \\
& +\frac{1}{[n]_{q}} \sum_{k=0}^{n-2}\left[\begin{array}{l}
n \\
k
\end{array}\right]_{q} q^{k}\left(\alpha_{n-k}-[n-k-1]_{q}[n-k]_{q} \beta_{n-k-2}\right)_{H} A_{k, q}(x), \quad n \geq 1 .
\end{aligned}
$$

Proof. Differentiation of generating function (15) with respect to $x$ and in view of fact that

$$
\frac{\partial^{k} G_{q}(x, t)}{\partial x^{k}}=t^{k} G_{q}(x, t),
$$

we have

$$
\sum_{n=0}^{\infty}{ }_{H} A_{n, q}(x) \frac{t^{n+k}}{[n]_{q} !}=D_{q, x}^{k}\left\{{ }_{H} A_{n, q}(x)\right\} \frac{t^{n}}{[n]_{q} !} .
$$

Comparing the coefficients of identical powers of $t$, we find

$$
D_{q, x H}^{k} A_{n, q}(x)=\frac{[n]_{q} !}{[n-k]_{q} !} H_{n-k, q}(x) .
$$


Since, the lowering operator $\Phi_{n, q}=\frac{1}{[n]_{q}} D_{q, x}$ satisfies operational rule:

$$
\Phi_{n, q H} A_{n, q}(x)={ }_{H} A_{n-1, q}(x),
$$

it follows that

$$
{ }_{H} A_{n-k, q}(x)=\left(\Phi_{n-k, q} \cdot \Phi_{n-k+1, q} \ldots \Phi_{n, q}\right)\left\{{ }_{H} A_{n, q}(x)\right\}=\frac{[n-k]_{q} !}{[n]_{q} !} D_{q, x}^{k}\left\{{ }_{H} A_{n, q}(x)\right\},
$$

which is derivative operator for $q$-HAP ${ }_{H} A_{n, q}(x)$.

Exchanging $x$ by $q x$ in Equation (15) and differentiating the obtained relation with respect to $t$ by making using of Formula (7), we get

$$
A_{q}(q t) F_{q}(q t) D_{q, t}\left(e_{q}(x q t)\right)+D_{q, t}\left(A_{q}(t) F_{q}(t)\right) e_{q}(x q t)=\sum_{n=0}^{\infty}{ }_{H} A_{n+1, q}(q x) \frac{t^{n}}{[n]_{q} !} .
$$

Again, using Formula (7) in Equation (29) and after that multiplying by $t$, it follows that

$$
\begin{gathered}
A_{q}(q t) F_{q}(q t) e_{q}(x q t) q x t+t D_{q, t}\left(A_{q}(t)\right) F_{q}(q t) e_{q}(x q t)+t A_{q}(t) D_{q, t}\left(F_{q}(t)\right) e_{q}(x q t) \\
=\sum_{n=0}^{\infty}[n]_{q H} A_{n, q}(q x) \frac{t^{n}}{[n] q !},
\end{gathered}
$$

which after simplifying gives

$$
\sum_{n=0}^{\infty}[n]_{q H} A_{n, q}(q x) \frac{t^{n}}{[n]_{q} !}=A_{q}(q t) F_{q}(q t) e_{q}(q x t)\left[q x t+\frac{t D_{q, t} A_{q}(t)}{A_{q}(q t)}+t \frac{D_{q, t} F_{q}(t)}{F_{q}(q t)} \frac{A_{q}(t)}{A_{q}(q t)}\right] .
$$

Using the facts that $t \frac{D_{q, t}\left(A_{q}(t)\right)}{A_{q}(q t)}=\sum_{n=0}^{\infty} \alpha_{n} \frac{t^{n}}{[n]_{q} !}, \frac{A_{q}(t)}{A_{q}(q t)}=\sum_{n=0}^{\infty} \beta_{n} \frac{t^{n}}{[n]_{q} !}$ and Equations (13) and (15) (with $t$ replaced by $q t$ ) the r.h.s. of above equation can be expressed as:

$$
\sum_{n=0}^{\infty}[n]_{q H} A_{n, q}(q x) \frac{t^{n}}{[n]_{q} !}=\sum_{n=0}^{\infty} q^{n} A_{n, q}(x) \frac{t^{n}}{[n]_{q} !}\left[q x t+\sum_{n=0}^{\infty} \alpha_{n} \frac{t^{n}}{[n]_{q} !}-t^{2} \sum_{n=0}^{\infty} \beta_{n} \frac{t^{n}}{[n]_{q} !}\right]
$$

which on simplification gives

$$
\begin{aligned}
\sum_{n=0}^{\infty}[n]_{q H} A_{n, q}(q x) \frac{t^{n}}{[n]_{q} !} & =\sum_{n=0}^{\infty}\left[[n]_{q} x q^{n}{ }_{H} A_{n-1, q}(x)+\sum_{k=0}^{n}\left[\begin{array}{l}
n \\
k
\end{array}\right]_{q} q^{n-k}{ }_{H} A_{n-k, q}(x) \alpha_{k}\right. \\
& \left.-\sum_{k=0}^{n-2}\left[\begin{array}{c}
n-2 \\
k
\end{array}\right]_{q}[n]_{q}[n-1]_{q} q^{n-k-2}{ }_{H} A_{n-k-2, q}(x) \beta_{k}\right] \frac{t^{n}}{[n]_{q} !} .
\end{aligned}
$$

Comparing the coefficients of identical powers of $t$ in above equation, we find

$$
\begin{aligned}
{ }_{H} A_{n, q}(q x) & =\frac{1}{[n]_{q}} q^{n} \alpha_{0 H} A_{n, q}(x)+q^{n}\left(x+\alpha_{1} q^{-1}\right)_{H} A_{n-1, q}(x) \\
& +\frac{1}{[n]_{q}} \sum_{k=2}^{n}\left[\begin{array}{l}
n \\
k
\end{array}\right]_{q} q^{n-k}\left(\alpha_{k}-[k]_{q}[k-1]_{q} \beta_{k-2}\right)_{H} A_{n-k, q}(x) .
\end{aligned}
$$

Replacing $k$ by $n-k$ in the third term of the r.h.s. of above equation, assertion (23) is proved.

Remark 3. Using Relation (28) in linear homogeneous recurrence Relation (34), the following consequence is deduced: 
Corollary 1. The q-Hermite-Appell polynomials ${ }_{H} A_{n, q}(x)$ satisfy the following $q$-difference equation:

$$
\left[\sum_{k=2}^{n} \frac{q^{n-k}}{[k]_{q} !}\left(\alpha_{k}-[k]_{q}[k-1]_{q} \beta_{k-2}\right) D_{q, x}^{k}+\left(q^{n}\left(x+\alpha_{1} q^{-1}\right)\right) D_{q, x}+\frac{q^{n} \alpha_{0}}{[0]_{q} !}\right]{ }_{H} A_{n, q}(x)-[n]_{q H} A_{n, q}(q x)=0 .
$$

Further, the $q$-recurrence relations and $q$-difference equations for several members of $q$-Hermite-Appell family are obtained by considering the following examples:

Example 1. Taking $A_{q}(t)=\left(\frac{t}{e_{q}(t)-1}\right)$ in Equation (15), we find

$$
\begin{aligned}
t \frac{D_{q, t}\left(A_{q}(t)\right)}{A_{q}(q t)} & =t \frac{D_{q, t} \frac{t}{e_{q}(t)-1}}{\frac{q t}{e_{q}(q t)-1}}=\sum_{n=0}^{\infty} \alpha_{n} \frac{t^{n}}{[n]_{q} !}, \\
\frac{A_{q}(t)}{A_{q}(q t)} & =\frac{\frac{t}{e_{q}(t)-1}}{\frac{q t}{e_{q}(q t)-1}}=\sum_{n=0}^{\infty} \beta_{n} \frac{t^{n}}{[n]_{q} !} .
\end{aligned}
$$

Using generating function of q-Bernoulli numbers (Table 1 (I)) and result ([16], p. 6 (24)-(25)) in $q$-recurrence Relation (23), the following linear homogeneous q-recurrence relation for $q$-HBP ${ }_{H} B_{n, q}(x)$ is obtained:

$$
\begin{aligned}
{ }_{H} B_{n, q}(q x) & =q^{n}\left(x-\frac{1}{[2]_{q}} q^{-1}\right){ }_{H} B_{n-1, q}(x)+\frac{1}{[n]_{q}} \sum_{k=0}^{n-2}\left[\begin{array}{l}
n \\
k
\end{array}\right]_{q} q^{k-1}\left(-b_{n-k, q}\right. \\
& \left.-[n-k-1]_{q}[n-k]_{q}(q-1) \sum_{l=0}^{n-k-2}\left[\begin{array}{l}
n-k-2 \\
l
\end{array}\right]_{q} b_{l, q}\right){ }_{H} B_{k, q}(x) .
\end{aligned}
$$

Similarly, we get the following $q$-difference equation for $q-H B P_{H} B_{n, q}(x)$ :

$$
\begin{aligned}
{\left[\sum_{k=2}^{n} \frac{q^{n-k-1}}{[k]_{q} !}(\right.} & \left.-b_{k, q}-[k]_{q}[k-1]_{q}(q-1) \sum_{l=0}^{k-2}\left[\begin{array}{c}
k-2 \\
l
\end{array}\right]_{q} b_{l, q}\right) D_{q, x}^{k} \\
& \left.+\left(q^{n}\left(x-\frac{1}{[2]_{q}} q^{-1}\right)\right) D_{q, x}\right]{ }_{H} B_{n, q}(x)-[n]_{q H} B_{n, q}(q x)=0 .
\end{aligned}
$$

Example 2. Taking $A_{q}(t)=\left(\frac{2}{e_{q}(t)+1}\right)$ in Equation (15), we get

$$
\begin{aligned}
t \frac{D_{q, t}\left(A_{q}(t)\right)}{A_{q}(q t)} & =t \frac{D_{q, t} \frac{2}{e_{q}(t)+1}}{\frac{2}{e_{q}(q t)+1}}=\sum_{n=0}^{\infty} \alpha_{n} \frac{t^{n}}{[n]_{q} !}, \\
\frac{A_{q}(t)}{A_{q}(q t)} & =\frac{\frac{2}{e_{q}(t)+1}}{\frac{2}{e_{q}(q t)+1}}=\sum_{n=0}^{\infty} \beta_{n} \frac{t^{n}}{[n]_{q} !} .
\end{aligned}
$$

Using generating function of $q$-Euler numbers (Table 1 (II)) and result ([16], p. 8 (32)-(33)) in q-recurrence Relation (23), the following linear homogeneous q-recurrence relation for $q-H E P{ }_{H} E_{n, q}(x)$ is obtained:

$$
\begin{aligned}
{ }_{H} E_{n, q}(q x) & =q^{n}\left(x-\frac{1}{2 q}\right){ }_{H} E_{n-1, q}(x)+\frac{1}{2[n]_{q}} \sum_{k=0}^{n-2}\left[\begin{array}{l}
n \\
k
\end{array}\right]_{q} q^{k}\left(E_{n-k-1, q}\right. \\
& \left.-[n-k-1]_{q}[n-k]_{q}(q-1) \sum_{l=0}^{n-k-2}\left[\begin{array}{c}
n-k-2 \\
l
\end{array}\right]_{q} E_{l, q}\right){ }_{H} E_{k, q}(x) .
\end{aligned}
$$


Also, the following q-difference equation for $q$-HEP ${ }_{H} E_{n, q}(x)$ is obtained:

$$
\begin{aligned}
{\left[\sum_{k=2}^{n} \frac{q^{n-k}}{2[k]_{q} !}\right.} & \left(E_{k-1, q}-[k]_{q}[k-1]_{q}(q-1) \sum_{l=0}^{k-2}\left[\begin{array}{c}
k-2 \\
l
\end{array}\right]_{q} E_{l, q}\right) D_{q, x}^{k} \\
& \left.+\left(q^{n}\left(x-\frac{1}{2 q}\right)\right) D_{q, x}\right]_{H} E_{n, q}(x)-[n]_{q H} E_{n, q}(q x)=0 .
\end{aligned}
$$

Example 3. Taking $A_{q}(t)=\left(\frac{2 t}{e_{q}(t)+1}\right)$ in Equation (15), we find

$$
\begin{aligned}
t \frac{D_{q, t}\left(A_{q}(t)\right)}{A_{q}(q t)} & =t \frac{D_{q, t} \frac{2 t}{e_{q}(t)+1}}{\frac{2 q t}{e_{q}(q t)+1}}=\sum_{n=0}^{\infty} \alpha_{n} \frac{t^{n}}{[n]_{q} !}, \\
\frac{A_{q}(t)}{A_{q}(q t)} & =\frac{\frac{2 t}{e_{q}(t)+1}}{\frac{2 q t}{e_{q}(q t)+1}}=\sum_{n=0}^{\infty} \beta_{n} \frac{t^{n}}{[n]_{q} !} .
\end{aligned}
$$

Using generating function of $q$-Genocchi numbers (Table 1 (III)) and result ([16], p. 9 (37) and (38)) in q-recurrence relation (23), the following linear homogeneous q-recurrence relation for $q$-HGP ${ }_{H} G_{n, q}(x)$ is obtained:

$$
\begin{aligned}
{ }_{H} G_{n, q}(q x) & =q^{n-1}{ }_{H} G_{n, q}(x)+q^{n}\left(x-\frac{1}{q^{2}}\right){ }_{H} G_{n-1, q}(x)+\frac{1}{2[n]_{q}} \sum_{k=0}^{n-2}\left[\begin{array}{l}
n \\
k
\end{array}\right]_{q} q^{k-1} \\
& \times\left(G_{n-k, q}-[n-k-1]_{q}[n-k]_{q}(q-1) \stackrel{\sum-k-2}{\left.\sum_{l=0}^{n-k-2}\left[\begin{array}{c}
n-2 \\
l
\end{array}\right]_{q} G_{l, q}\right){ }_{H} G_{k, q}(x) .}\right.
\end{aligned}
$$

Also, the following q-difference equation for $q-H G P{ }_{H} G_{n, q}(x)$ is obtained:

$$
\begin{aligned}
& {\left[\sum_{k=2}^{n} \frac{q^{n-k-1}}{2[k]_{q} !}\left(G_{k, q}-[k]_{q}[k-1]_{q}(q-1) \sum_{l=0}^{k-2}\left[\begin{array}{c}
k-2 \\
l
\end{array}\right]_{q} G_{l, q}\right) D_{q, x}^{k}\right.} \\
& \left.\quad+\left(q^{n}\left(x-\frac{1}{q^{2}}\right)\right) D_{q, x}+q^{n-1}\right]{ }_{H} G_{n, q}(x)-[n]_{q H} G_{n, q}(q x)=0 .
\end{aligned}
$$

In the next section, the graphs of $q$-HBP ${ }_{H} B_{n, q}(x), q$-HEP ${ }_{H} E_{n, q}(x)$ and $q$-HGP ${ }_{H} G_{n, q}(x)$ are displayed by using Matlab (R2010a, Mathworks, New Delhi, India). The zeros of these polynomials are also investigated using numerical computations.

\section{Concluding Remarks}

Nowadays, there has been progressively instantaneous development in the computing environment. The numerical investigation is very much essential to understand the basic concepts of $q$-numbers and $q$-polynomials. By using computer software, we can examine concepts much more easily. In 2006, Ryoo et al. [17] provide the numerical investigation of the zeros of certain $q$-polynomials. Moreover, the graphs of a new class of $q$-Bernoulli polynomials are explored in [18]. Research in this direction will lead to a captivating approach that employs numerical methods in the fields of these $q$-special polynomials to turn out in many areas of mathematics and mathematical physics.

First, we plot the graphs of $q$-HBP ${ }_{H} B_{n, q}(x), q$-HEP ${ }_{H} E_{n, q}(x)$ and $q$-HGP ${ }_{H} G_{n, q}(x)$ for $n=1,2,3,4$ and $q=\frac{1}{2}(0<q<1)$. The zeros of these $q$-special polynomials are also investigated and their behaviour is observed. This will show the four plots combined into one for each of these polynomials.

To show graphical representation of $q$ - $\mathrm{HBP}_{H} B_{n, q}(x), q-\mathrm{HEP}{ }_{H} E_{n, q}(x)$ and $q$-HGP ${ }_{H} G_{n, q}(x)$, the values of first five $B_{n, q}(x), E_{n, q}(x), G_{n, q}(x)$ and $H_{n, q}(x)$ are required. Using the values listed in 
Table 2, the expressions of first five $B_{n, q}(x), E_{n, q}(x), G_{n, q}(x)$ and $H_{n, q}(x)$ are obtained and are mentioned in Table 5.

Table 5. Expressions of first five $B_{n, q}(x), E_{n, q}(x), G_{n, q}(x)$ and $H_{n, q}(x)$.

\begin{tabular}{|c|c|c|c|c|c|}
\hline$n$ & 0 & 1 & 2 & 3 & 4 \\
\hline$B_{n, q}(x)$ & 1 & $x-\frac{1}{1+q}$ & $\begin{array}{l}x^{2}-\frac{[2]_{q}}{1+q} x \\
+\frac{q^{2}}{[3]_{q}[2]_{q}}\end{array}$ & $x^{3}-\frac{[3]_{q} x^{2}}{1+q}+\frac{q^{2} x}{[2]_{q}}+\frac{(1-q) q^{3}}{[2]_{q}[4]_{q}}$ & $\begin{array}{c}x^{4}-\frac{[4]_{q}}{1+q} x^{3}+\frac{[4]_{q} q^{2}}{[2]_{q}^{2}} x^{2}+\frac{(1-q) q^{3}}{[2]_{q}} x \\
+\frac{q^{4}\left(1-q^{2}-2 q^{3}-q^{4}+q^{6}\right)}{\left([2]_{q}^{2}[3]_{q}[5]_{q}\right)}\end{array}$ \\
\hline$E_{n, q}(x)$ & 1 & $x-\frac{1}{2}$ & $\begin{aligned} & x^{2}-\frac{[2]_{q}}{2} x \\
+ & \frac{1}{4}(-1+q)\end{aligned}$ & $\begin{array}{c}x^{3}-\frac{[3]_{q}}{2} x^{2}+\frac{[3]_{q}}{4}(-1+q) x \\
+\frac{1}{8}\left(-1+2 q+2 q^{2}-q^{3}\right)\end{array}$ & $\begin{array}{c}x^{4}-\frac{[4]_{q}}{2} x^{3}+\frac{[4]_{q}[3]_{q}(q-1)}{4[2]_{q}} x^{2}+ \\
\frac{[4]_{q}\left(-1+2 q+2 q^{2}-q^{3}\right)}{8} x \\
+\frac{(q-1)[3]_{q} !\left(q^{2}-4 q+1\right)}{16}\end{array}$ \\
\hline$G_{n, q}(x)$ & 0 & $\frac{2-q}{1+q}$ & $(2-q) x+\frac{q(q-5)}{(1+q)^{2}}$ & $\begin{array}{c}{[3]_{q} \frac{(2-q)}{(1+q)} x^{2}+[3]_{q} \frac{q(q-5)}{(1+q)^{2}} x} \\
-\frac{q}{1+q}-[3]_{q} \frac{q^{2}(q-5)}{(1+q)^{3}}-\frac{3 q(2-q)}{(1+q)^{2}}\end{array}$ & $\begin{array}{c}{[4]_{q} \frac{(2-q)}{(1+q)} x^{3}+[4]_{q}[3]_{q} \frac{q(q-5)}{(1+q)^{3}} x^{2}} \\
+[4]_{q} \frac{\left(-q^{3} 10 q^{2}-7 q\right)}{(1+q)^{3}} x \\
-\frac{3 q}{1+q}\left(\frac{3 q^{3}+10 q^{2}-28 q+7}{(1+q)^{3}}\right)\end{array}$ \\
\hline$H_{n, q}(x)$ & 1 & $x$ & $x^{2}-1$ & $x^{3}-[3]_{q} x$ & $x^{4}-\left(1+q^{2}\right)[3]_{q} x^{2}+[3]_{q} q^{2}$ \\
\hline
\end{tabular}

Further, by making appropriate substitutions from Tables 2 and 5 in series definitions mentioned in Table 3 (I-III), the expressions of the first five $q-\mathrm{HBP}_{H} B_{n, q}(x), q-\mathrm{HEP}_{H} E_{n, q}(x)$ and $q-\mathrm{HGP}_{H} G_{n, q}(x)$ for $q=\frac{1}{2}$ are obtained and are mentioned in Table 6 .

Table 6. Expressions of first five ${ }_{H} B_{n, 1 / 2}(x),{ }_{H} E_{n, 1 / 2}(x)$ and ${ }_{H} G_{n, 1 / 2}(x)$.

\begin{tabular}{cccccc}
\hline $\boldsymbol{n}$ & $\mathbf{0}$ & $\mathbf{1}$ & $\mathbf{2}$ & $\mathbf{3}$ & $\mathbf{4}$ \\
\hline${ }_{H} B_{n, 1 / 2}(x)$ & 1 & $x-\frac{2}{3}$ & $x^{2}-x-\frac{19}{21}$ & $x^{3}-\frac{7}{6} x^{2}-\frac{19}{12} x+\frac{107}{90}$ & $x^{4}-\frac{5}{4} x^{3}-\frac{95}{48} x^{2}-\frac{13}{48} x-\frac{3197}{15624}$ \\
\hline${ }_{H} E_{n, 1 / 2}(x)$ & 1 & $x-\frac{1}{2}$ & $x^{2}-\frac{3}{4} x-\frac{9}{8}$ & $x^{3}-\frac{63}{32} x-\frac{7}{8} x^{2}+\frac{59}{64}$ & $x^{4}-\frac{15}{16} x^{3}-\frac{315}{128} x^{2}+\frac{885}{512} x+\frac{665}{1024}$ \\
\hline${ }_{H} G_{n, 1 / 2}(x)$ & 0 & 1 & $\frac{3}{2} x-1$ & $\frac{7}{4} x^{2}+\frac{7}{4} x-\frac{25}{12}$ & $\frac{15}{8} x^{3}-\frac{35}{16} x^{2}-\frac{125}{32} x+\frac{491}{144}$ \\
\hline $\begin{array}{l}\text { Note: From Table 6, it can be seen that degree of } q \text {-Hermite-Genocchi polynomials is } n-1 \text {. Hence, }{ }_{H} G_{n, q}(x) \text { is } \\
\text { considered in the class of polynomial sequences which are not in } q \text {-Hermite-Appell family in strong sense. }\end{array}$
\end{tabular}

Using Matlab and by using the expressions of ${ }_{H} B_{n, 1 / 2}(x){ }_{H} E_{n, 1 / 2}(x)$ and ${ }_{H} G_{n, 1 / 2}(x)$ from Table 6 for $n=1,2,3,4$, Figure 1 is drawn.

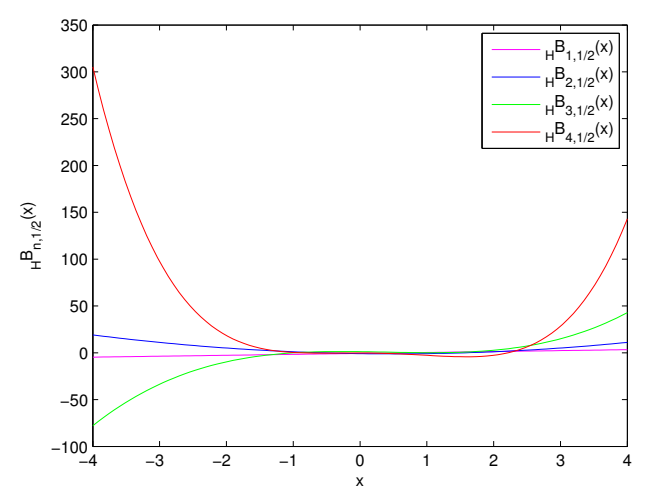

(a)

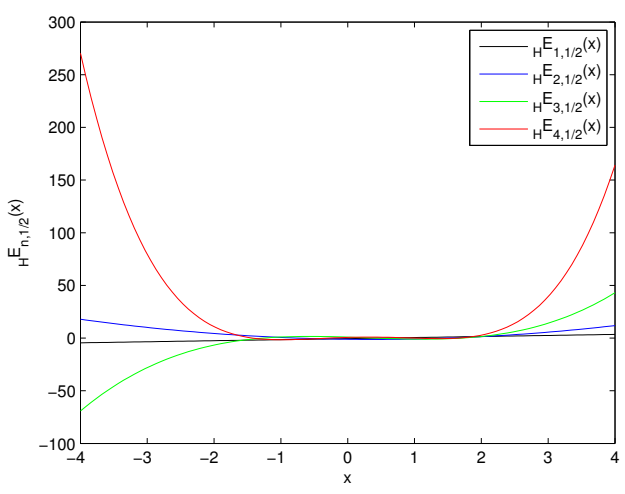

(b)

Figure 1. Cont. 


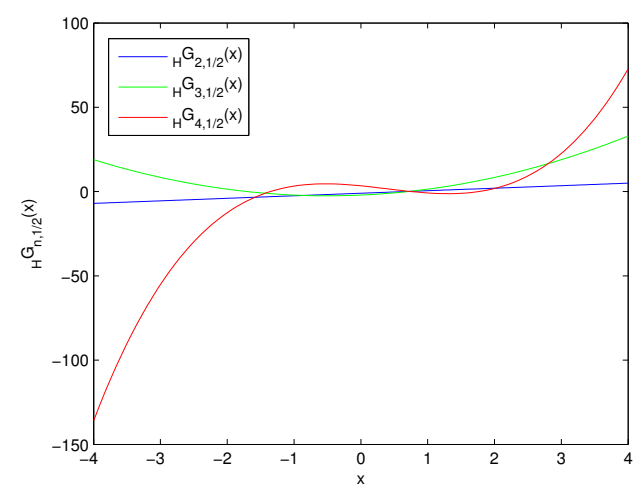

(c)

Figure 1. (a) Shape of ${ }_{H} B_{n, 1 / 2}(x) ;(\mathbf{b})$ Shape of ${ }_{H} E_{n, 1 / 2}(x) ;(\mathbf{c})$ Shape of ${ }_{H} G_{n, 1 / 2}(x)$.

Next, the real and complex zeros of $q$-HBP ${ }_{H} B_{n, 1 / 2}(x), q$-HEP ${ }_{H} E_{n, 1 / 2}(x)$ and $q$-HGP ${ }_{H} G_{n, 1 / 2}(x)$ are computed using Matlab for same values of $n, q$ and $x \in \mathbb{C}$. These are given in Tables 7 and 8 .

Table 7. Real zeros of ${ }_{H} B_{n, 1 / 2}(x),{ }_{H} E_{n, 1 / 2}(x)$ and ${ }_{H} G_{n, 1 / 2}(x)$.

\begin{tabular}{cccc}
\hline Degree $n$ & ${ }_{H} \boldsymbol{B}_{\boldsymbol{n}, \mathbf{1 / 2}}(\boldsymbol{x})$ & ${ }_{\boldsymbol{H}} \boldsymbol{E}_{\boldsymbol{n}, \mathbf{1} / \mathbf{2}}(\boldsymbol{x})$ & ${ }_{H} \boldsymbol{G}_{\boldsymbol{n}, \mathbf{1 / 2}}(\boldsymbol{x})$ \\
\hline 1 & 0.6667 & 0.5000 & 0 \\
2 & $-0.5746,1.5746$ & $-0.7500,1.5000$ & 0.6667 \\
3 & $-1.1392,0.6184,1.6874$ & $-1.2626,0.4268,1.7108$ & $-1.7002,0.7002$ \\
4 & $-0.8987,2.2167$ & $-1.4220,-0.2797,0.9898,1.6494$ & $-1.3568,1.7633,0.7601$ \\
\hline
\end{tabular}

Table 8. Complex zeros of ${ }_{H} B_{n, 1 / 2}(x),{ }_{H} E_{n, 1 / 2}(x)$ and ${ }_{H} G_{n, 1 / 2}(x)$.

\begin{tabular}{cccc}
\hline Degree $n$ & ${ }_{H} B_{n, \mathbf{1} / \mathbf{2}}(x)$ & ${ }_{H} E_{n, \mathbf{1} / \mathbf{2}}(x)$ & ${ }_{H} G_{n, \mathbf{1} / \mathbf{2}}(x)$ \\
\hline 1 & - & - & - \\
2 & - & - & - \\
3 & - & - & - \\
4 & $-0.0340+0.3187 i,-0.0340-0.3187 i$ & - & - \\
\hline
\end{tabular}

Remark 4. In view of the values given in Tables 7 and 8, the following important relation between the real and complex zeros of $q-H A P_{H} A_{n, q}(x)$ is observed:

Number of $\left\{\right.$ Real zeros of $\left.{ }_{H} A_{n, q}(x)\right\}=n-$ Number of $\left\{\right.$ Complex zeros of $\left.{ }_{H} A_{n, q}(x)\right\}$, where $n$ is degree of the polynomial.

Further, we plot the distribution of zeros of ${ }_{H} B_{n, 1 / 2}(x),{ }_{H} E_{n, 1 / 2}(x)$ and ${ }_{H} G_{n, 1 / 2}(x)$ for $n=1,2,3,4$ in Figure 2. 


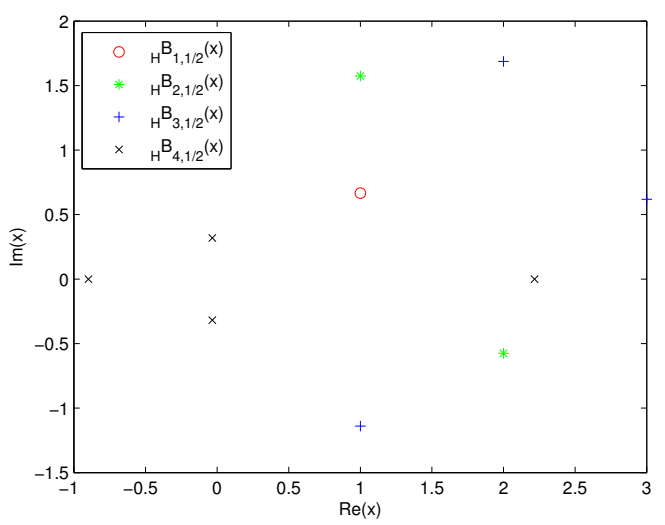

(a)

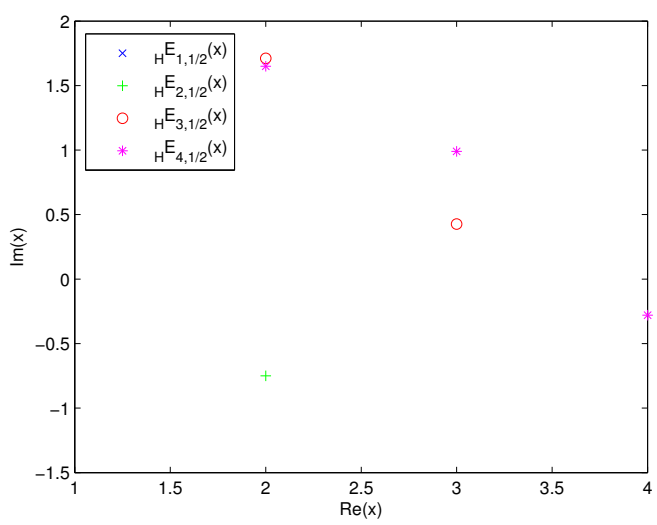

(b)

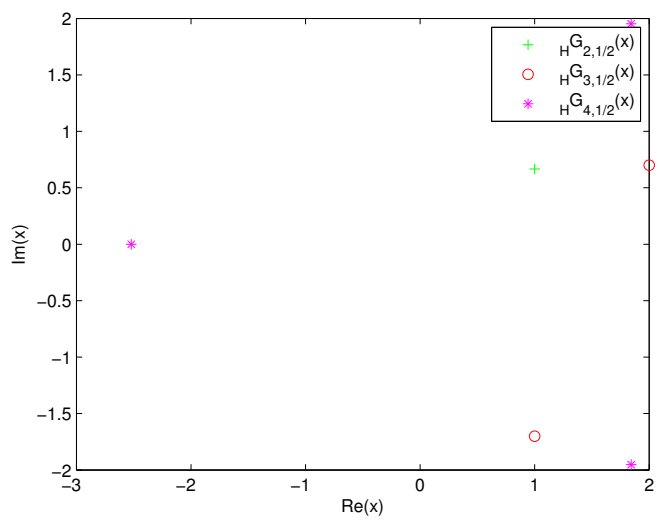

(c)

Figure 2. (a) Zeros of ${ }_{H} B_{n, 1 / 2}(x)$; (b) Zeros of ${ }_{H} E_{n, 1 / 2}(x)$; (c) Zeros of ${ }_{H} G_{n, 1 / 2}(x)$.

In order to make the above discussion more clear, we draw the combined graphs of shape and zeros of ${ }_{H} B_{n, 1 / 2}(x){ }_{H} E_{n, 1 / 2}(x)$ and ${ }_{H} G_{n, 1 / 2}(x)$ for $n=4$ in Figures 3 and 4 .

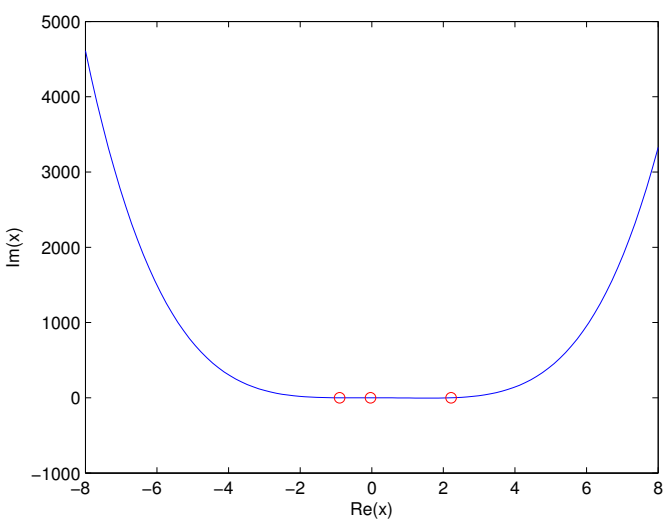

Figure 3. Shape and zeros of ${ }_{H} B_{4,1 / 2}(x)$. 

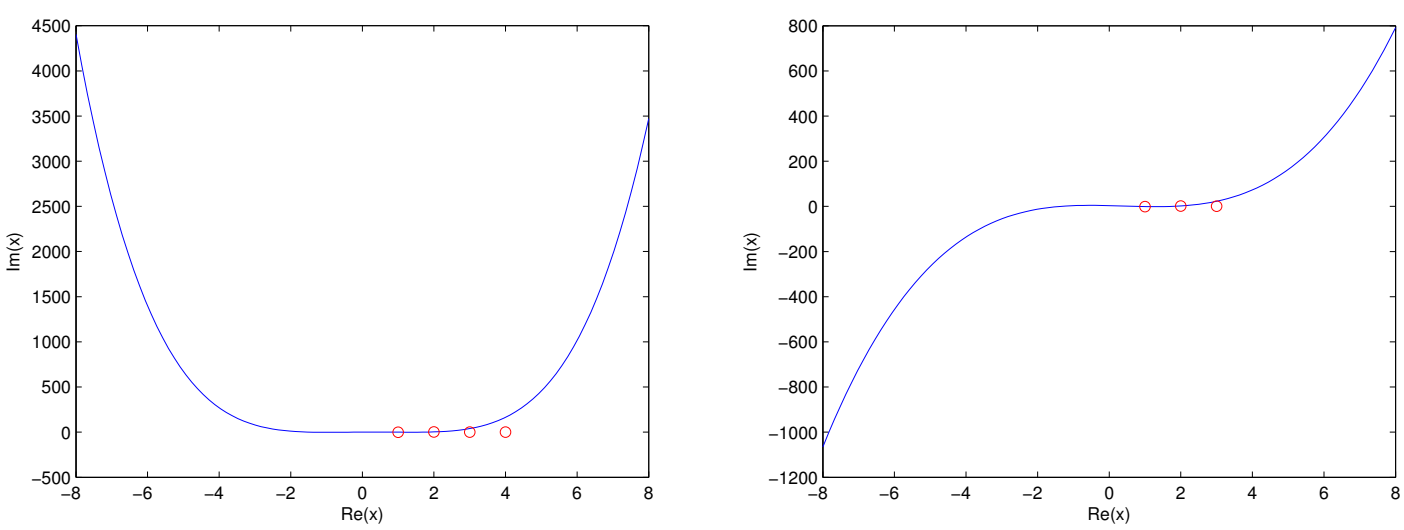

Figure 4. Shape and zeros of ${ }_{H} E_{4,1 / 2}(x)$, Shape and zeros of ${ }_{H} G_{4,1 / 2}(x)$.

It is to be noted that in Figure 3 out of total two complex zeros only one, with positive imaginary part is visible, due to the absence of negative imaginary axis in this graphs.

These figures gives mathematicians an unrestricted capability to create visual mathematical investigations of the behaviour of $q$-HAP ${ }_{H} A_{n, q}(x)$. The approach presented in this paper is general and opens new possibilities to deal with other hybrid families of $q$-special polynomials. The results established in this article may find applications in certain branches of mathematics, physics and engineering.

The following Appendix A aims to demonstrate the shape and zeros of $q$-HBP ${ }_{H} B_{n, q}(x)$ for $q=\frac{1}{5}$.

Author Contributions: Supervision, S.K.; Writing-original draft, T.N.

Funding: This work has been done under Senior Research Fellowship (Award letter No. F./2014-15/NFO-201415-OBC-UTT-24168/(SA-III/Website)) awarded to Tabinda Nahid by the University Grants Commission, Government of India, New Delhi.

Conflicts of Interest: The authors declare no conflict of interest.

\section{Appendix A}

To show graphical representation of $q$-HBP ${ }_{H} B_{n, q}(x)$ for $n=1,2,3,4$ and $q=\frac{1}{5}$, first few expressions of $q$-HBP ${ }_{H} B_{n, 1 / 5}(x)$ are obtained by making appropriate substitutions from Tables 2 and 5 in series definitions mentioned in Table 3 (I). These are mentioned in Table A1.

Table A1. Expressions of first five ${ }_{H} B_{n, 1 / 5}(x)$.

\begin{tabular}{cccccc}
\hline $\boldsymbol{n}$ & $\mathbf{0}$ & $\mathbf{1}$ & $\mathbf{2}$ & $\mathbf{3}$ & $\mathbf{4}$ \\
\hline${ }_{H} B_{n, 1 / 5}(x)$ & 1 & $x-\frac{5}{6}$ & $x^{2}-x-\frac{181}{186}$ & $x^{3}-\frac{31}{30} x^{2}-\frac{181}{150} x+\frac{3349}{3120}$ & $x^{4}-\frac{26}{25} x^{3}-\frac{2353}{1875} x^{2}+\frac{2428}{1875} x+\frac{4254523}{272373750}$ \\
\hline
\end{tabular}

Next, the real and complex zeros of $q-\mathrm{HBP}_{H} B_{n, 1 / 5}(x)$ are computed using Matlab for same values of $n, q$ and $x \in \mathbb{C}$. These are given in Table A2.

Table A2. Zeros of ${ }_{H} B_{n, 1 / 5}(x)$.

\begin{tabular}{cc}
\hline Degree $n$ & $\boldsymbol{H}_{\boldsymbol{n}, \mathbf{1} / \mathbf{5}}(\boldsymbol{x})$ \\
\hline 1 & 0.833333 \\
2 & $0.605947,1.60595$ \\
3 & $1.3349-6.3159 \times 10^{-16} i, 0.75822+5.55112 \times 10^{-16} i,-1.06009-1.38778 \times 10^{-16_{i}}$ \\
4 & $1.08357+0.0177105 i, 1.08357-0.0177105 i,-1.11521,-0.0119261$ \\
\hline
\end{tabular}

With the help of Matlab and using the values from Tables A1 and A2, the foregoing Figure A1 is drawn. 


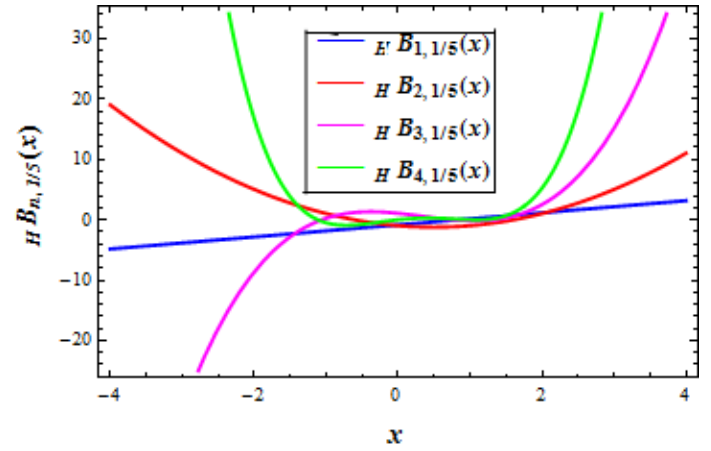

(a)

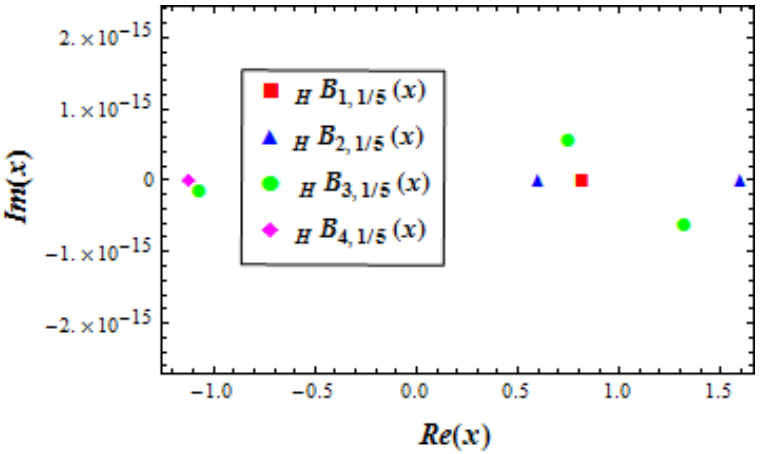

(b)

Figure A1. (a) Shape of ${ }_{H} B_{n, 1 / 5}(x)$; (b) Zeros of ${ }_{H} B_{n, 1 / 5}(x)$.

The structure properties of a particular class of normed quasi *-algebras called $C Q^{*}$-algebras are studied in [19-21]. It is important to remark that quasi ${ }^{*}$-algebras provide an appropriate mathematical framework for discussing certain quantum physical systems for which the usual algebraic approach is not sufficient. The possibility of extending the Gelfand-Naimark representation of $C^{*}$-algebras to certain Banach $C^{*}$-modules is considered by S. Triolo [22]. The existence of modular representations and their continuity were studied in $[23,24]$, while a special class of Banach modules over a $W Q^{*}$-algebra is considered in [22] with certain conditions for the existence of a faithful representation.

\section{References}

1. Andrews, G.E.; Askey, R.; Roy, R. Special Functions, Encyclopedia of Mathematics and Its Applications; Cambridge University Press: Cambridge, UK, 1999; Volume 71.

2. Andrews, G.E.; Askey, R. Classical orthogonal polynomials. In Polynômes Orthogonaux et Applications; Lecture Notes in Mathematics; Springer: Berlin, Germany, 1984; Volume 1171, pp. 36-63.

3. Sharma, K.; Jain, R. Lie theory and q-Appell functions. Proc. Natl. Acad. Sci. India Sect. A Phys. Sci. 2007, 77, 259-261.

4. Ernst, T. Convergence aspects for $q$-Appell functions. Proc. Natl. Acad. Sci. India Sect. A Phys. Sci. 2014, 81, 67-77.

5. Appell, P. Sur une classe de polynômes. Ann. Sci. Ėcole Norm. Sup. 1880, 9, 119-144. [CrossRef]

6. Al-Salam, W.A. q-Appell polynomials. Ann. Mat. Pura Appl. 1967, 4, 31-45. [CrossRef]

7. Al-Salam, W.A. q-Bernoulli numbers and polynomials. Math. Nachr. 1959, 17, 239-260. [CrossRef]

8. Ernst, T. q-Bernoulli and q-Euler polynomials, an Umbral approach. Int. J. Differ. Equ. 2006, 1, 31-80.

9. Mahmudov, N.I. On a class of $q$-Bernoulli and $q$-Euler polynomials. Adv. Differ. Equ. 2013, 108, 1-11. [CrossRef]

10. Araci, S.; Acikgoz, M.; Jolany, H.; He, Y. Identities involving $q$-Genocchi numbers and polynomials. Notes Number Theory Discret. Math. 2014, 20, 64-74.

11. Mahmudov, N.I. Difference equations of $q$-Appell polynomials. Appl. Math. Comput. 2014, 245, 539-543. [CrossRef]

12. Srivastava, H.M. Some characterizations of Appell and q-Appell polynomials. Ann. Mat. Pura Appl. 1982, 4, 321-329.

13. Loureiro, A.F; Maroni, P. Around q-Appell polynomials sequences. Ramanujan J. 2011, 26, 311-321. [CrossRef]

14. Keleshteri, M.E.; Mahmudov, N.I. A study on $q$-Appell polynomials from determinantal point of view. Appl. Math. Comput. 2015, 260, 351-369. [CrossRef]

15. He, M.X.; Ricci, P.E. Differential equation of Appell polynomials via the factorization method. J. Comput. Appl. Math. 2002, 139, 231-237. [CrossRef]

16. Keleshteri, M.E.; Mahmudov, N.I. On the class of 2D q-Appell polynomials. arXiv 2015, arXiv:1512.03255v1. 
17. Ryoo, C.S.; Kim, T.; Agarwal, R.P. A numerical investigation of the roots of $q$-polynomials. Int. J. Comput. Math. 2006, 83, 223-234. [CrossRef]

18. Ryoo, C.S. A note on $q$-Bernoulli numbers and polynomials. Appl. Math. Lett. 2007, 20, 524-531. [CrossRef]

19. Antoine, J.-P.; Inoue, A.; Trapani, C. Partial *-Algebras and their Opertaor Realizations; Kluwer: Dordrecht, The Netherlands, 2002.

20. Bagarello, F.; Trapani, C.; Triolo, S. Quasi *-algebras of measurable operators. Studia Math. 2006, 172, 289-305. [CrossRef]

21. Takesaki, M. Theory of Operator Algebras I; Springer: New York, NY, USA, 1979.

22. Triolo, S. WQ*-algebras of measurable operators. Indian J. Pure Appl. Math. 2012, 43, 601-617. [CrossRef]

23. Trapani, C.; Triolo, S. Representations of certain Banach $C^{*}$-modules. Mediterranean J. Math. 2004, 1, 441-461. [CrossRef]

24. Trapani, C.; Triolo, S. Representations of modules over a *-algebra and related seminorms. Studia Math. 2008, 184, 133-148. [CrossRef]

(c) 2018 by the authors. Licensee MDPI, Basel, Switzerland. This article is an open access article distributed under the terms and conditions of the Creative Commons Attribution (CC BY) license (http:/ / creativecommons.org/licenses/by/4.0/). 